\title{
Polyhedral water droplets: shape transitions
}

\section{and mechanism}

\section{Supporting Information}

Shir R. Liber ${ }^{\dagger}$ Orlando Marin, ${ }^{\dagger}$ Alexander V. Butenko, ${ }^{\dagger}$ Racheli Ron, ${ }^{\ddagger}$ Lee Shool ${ }^{\dagger}$ Adi Salomon, ${ }^{\ddagger}$ Moshe Deutsch,${ }^{\dagger}$ and Eli Sloutskin ${ }^{* \dagger}$

$\dagger$ Physics Dept. \& Institute of Nanotechnology and Advanced Materials, Bar-Ilan University, Ramat-Gan 52900, Israel

$\ddagger$ Chemistry Dept. \& Institute of Nanotechnology and Advanced Materials, Bar-Ilan University, Ramat-Gan 52900, Israel

E-mail: eli.sloutskin@biu.ac.il 


\section{Supporting Materials and Methods}

\subsection{Sample preparation}

\subsubsection{Materials}

The emulsions studied consisted of water, a surfactant, and an oil. Millipore Ultrapure $18.2 \mathrm{M} \Omega \cdot \mathrm{cm}$ water was used throughout. The surfactant, octadecyltrimethylamonium bromide $\left(\mathrm{C}_{18} \mathrm{TAB}\right)$ (SigmaAldrich, 98\% pure), was recrystallized twice from a methanol - acetone solution, then twice recrystallized from a hexane-acetone solution. The oil, hexadecane $\left(\mathrm{C}_{16}\right)$ (Aldrich, $99 \%$ pure), was percolated 2-3 times through a freshly activated basic alumina powder column to remove acidic polar impurities. For hexadecane-alcohol mixtures, hexadecanol $\left(\mathrm{C}_{16} \mathrm{OH}\right)$ (Aldrich, $99 \%$ pure) was used as received. For confocal imaging of the oil droplets in oil-in-water emulsions, the oil was labeled by the hydrophobic Bodipy ${ }^{T M} 505 / 515$ (D3921) dye. For confocal imaging of the water

droplets in water-in-oil emulsions, the water was labeled by the hydrophilic Bodipy ${ }^{T M} 492 / 515$ (D3238) dye, used as received.

\subsubsection{Stock solutions' preparation}

For forming the emulsions, we first prepare a $1 \mathrm{mM}$ water solution of the $\mathrm{C}_{18} \mathrm{TAB}$. The solution was stirred for $>40$ minutes at $50^{\circ} \mathrm{C}$, ensuring a complete dissolution of the surfactant. When a $\mathrm{C}_{16}: \mathrm{C}_{16} \mathrm{OH}$ mixture was used, it was premixed by magnetic stirring on a hot-plate, at $T>$ $60^{\circ} \mathrm{C}$, well above the melting point of $\mathrm{C}_{16} \mathrm{OH}$. For the optical microscopy studies of the water:oil meniscus the $\mathrm{C}_{16} \mathrm{OH}$ mass concentration was $0.4 \%$, the highest concentration explored by our interfacial tension measurements (Figure 2 of the main text). Since the total interfacial area of the droplets in an emulsion is much higher than that of the meniscus between bulks, we varied the $\mathrm{C}_{16} \mathrm{OH}$ concentration in the oil phase of our emulsions between $0.4 \%$ and $1.3 \%$. No qualitative difference in the behavior of the droplet shapes, or in any other aspect of the emulsions' behavior, was observed within this range of concentrations. However, $T_{d}$ in the $1.3 \% \mathrm{C}_{16} \mathrm{OH}$ samples was 
higher by $\sim 6^{\circ} \mathrm{C}$ than that in the $0.4 \%$ samples. No dye was added to most of the samples, to minimize contaminations and radiation-induced formation of free radicals.

\subsubsection{Emulsion samples' preparation and loading}

Emulsion samples were prepared by introducing $1-2 \%$ (by weight) of the dispersed phase into the suspending phase, followed by $\sim 2$ min mixing at $T>T_{s}$. The emulsion was then loaded by capillary rise into borosilicate Vitrocom $\mathbb{R} \quad 0.1 \times 2 \times 50 \mathrm{~mm}, 0.2 \times 2 \times 50 \mathrm{~mm}$, or $0.1 \times 1 \times 50 \mathrm{~mm}$ rectangular glass capillaries and sealed by an instant Epoxy glue. No systematic dependence of any of the results on the capillary dimensions was detected. To minimize the distortion of water-in-oil droplets due to wetting effects at the capillary walls, the capillaries used in these experiments have been hydrophobized prior to sample loading. No capillary-wetting related issues were observed for the oil-in-water emulsions. Thus, untreated capillaries were used for these emulsions. ${ }^{1}$

\subsubsection{Planar-interface samples' preparation and loading}

To form an oil:water quasi-planar interface, we load the aqueous phase into a Vitrocom ${ }^{T M}$ capillary by capillary action, filling half of the capillary length. Next, the same end of the capillary is dipped into the oil phase. The capillarity-driven flow of oil into the capillary pushes the aqueous solution upwards, allowing the whole volume of the capillary to be filled. The oil-water interface, thus located in the center of the capillary, is clearly visible by the unaided eye. To allow imaging the full shape of the planar interface by high-resolution confocal microscopy, a square $0.2 \times 0.2 \times 50 \mathrm{~mm}^{3}$ Vitrocom $^{T M}$ capillary was employed in Fig. 4B (main text), instead of the rectangular ones. The flows involved in the preparation of the planar interface samples cause the formation of oil-in-water and water-in-oil emulsion droplets on the corresponding sides of the interface, allowing the emulsion studies to be carried out in the same sample. The behavior of the emulsion droplets prepared by this procedure was indistinguishable from that of the emulsion droplets prepared by bulk mixing. 


\subsubsection{Temperature control}

Once filled with the sample, the capillary was glued with its wide face down onto a brass slide, having a narrow machined opening for optical observations. The slide is inserted horizontally into a home-made temperature-controlled cell, mounted on the translation stage of an inverted microscope. The sample cell employs a pair of Peltier elements for baseplate cooling, and a Lakeshore model 330 for PID temperature control via thin-film resistive heaters and a $100 k \Omega$ precision thermistor. Our setup allows the temperature of the sample to be regulated to $0.01{ }^{\circ} \mathrm{C}$, in the range from 10 to $60{ }^{\circ} \mathrm{C}$.

\subsection{Capillaries' hydrophobization}

While the faceting of water-in-oil droplets is visible even for samples contained in the as-received borosilicate Vitrocom ${ }^{T M}$ capillaries, the wetting effects at the hydrophilic boundaries of these capillaries strongly distort the droplets. Also, no icosahedra are formed, and only platelet-like shapes are observed. To minimize the boundary-wetting effects, we hydrophobized the glass capillaries prior to use.

For the hydrophobization, we first washed the capillaries by a $1 \%$ Hellmanex $^{T M}$ solution in Millipore Ultrapure water and sonicated them in this solution at $40^{\circ} \mathrm{C}$, for 20 minutes. Next, the capillaries were washed by ethanol and dried under nitrogen flow (99.999\% purity). The dried capillaries were then soaked for $18 \mathrm{hrs}$ in a $2 \%$ chlorotrimethylsilane solution in anhydrous hexane and anhydrous ethanol (1:1). This solution was prepared by fully dissolving the chlorotrimethylsilane in hexane, then adding slowly the ethanol. Finally, the capillaries were rinsed by anhydrous ethanol and thoroughly dried under a pure nitrogen flow.

This hydrophobization procedure significantly reduces the wetting at the boundaries of the capillary, so that the icosahedral shapes are clearly observed (Fig. S1; see also Fig. 1C of the main text). However, the hydrophobic layer is not totally defect-free. Assuming the distribution of such defects to be random, the probability for a radius- $R$ droplet to be located at a defect scales as 


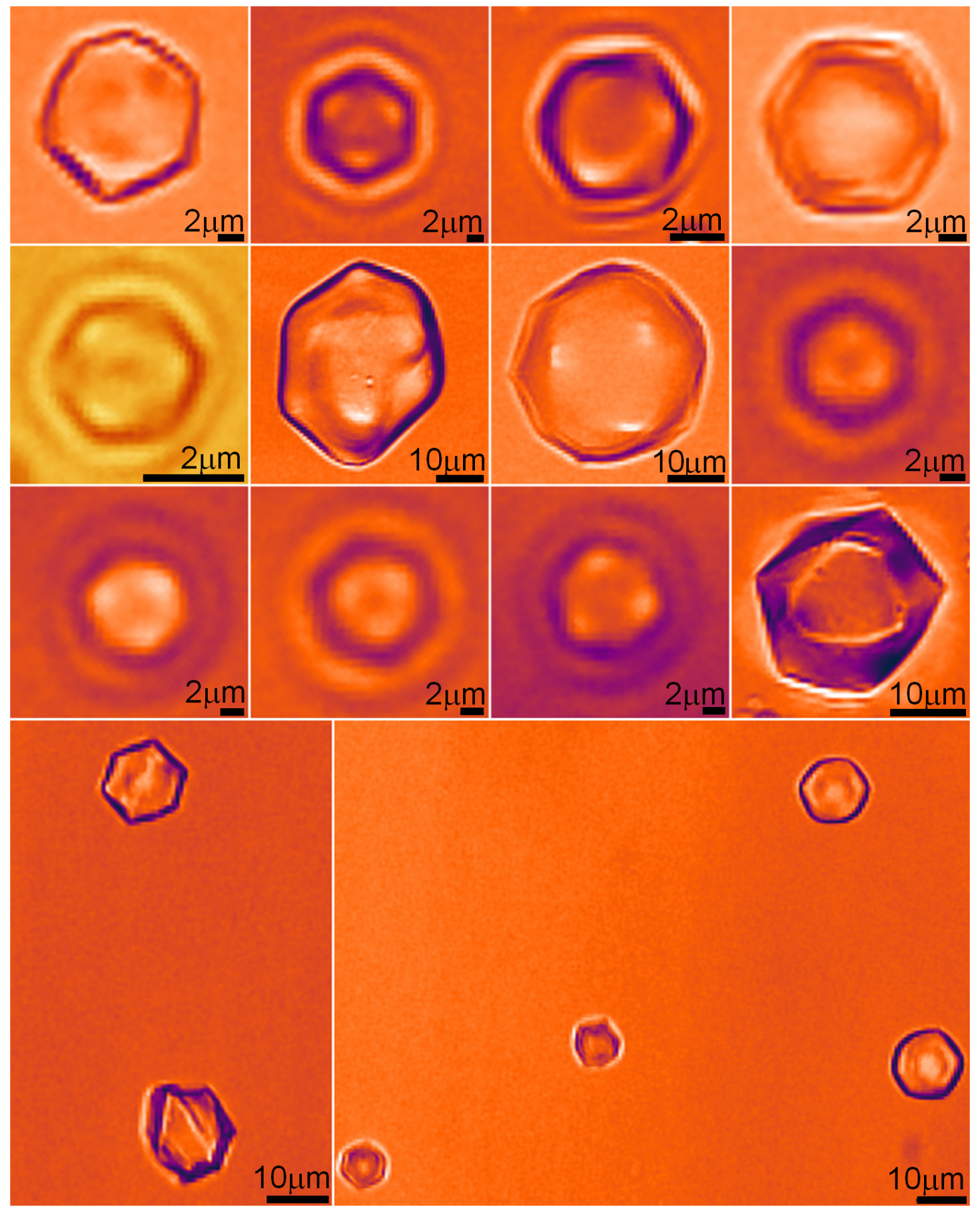

Figure S1: Water-in-oil icosahedral droplets. Aqueous $\mathrm{C}_{18} \mathrm{TAB}(1 \mathrm{mM})$ solution droplets, dispersed in $\mathrm{C}_{16}$ oil, laced with $\mathrm{C}_{16} \mathrm{OH}$ (0.4-1.3\%), just below the sphere-to-icosahedral transition. Note the higher-perfection, less distorted, icosahedral shapes of the small droplets. The larger droplets are more strongly distorted, as they are more likely to touch one of the defects in the capillary's hydrophobic coating. 
$R^{2}$. Thus, the larger droplets are more likely to be distorted by glass-wetting effects. The greater distortion of the large droplets, compared to the small ones, is indeed observed in Fig. S1. Finally, on further cooling, the icosahedra adopt faceted platelet-like shapes, as demonstrated in Fig. S2 (see also Fig. 1D of the main text).

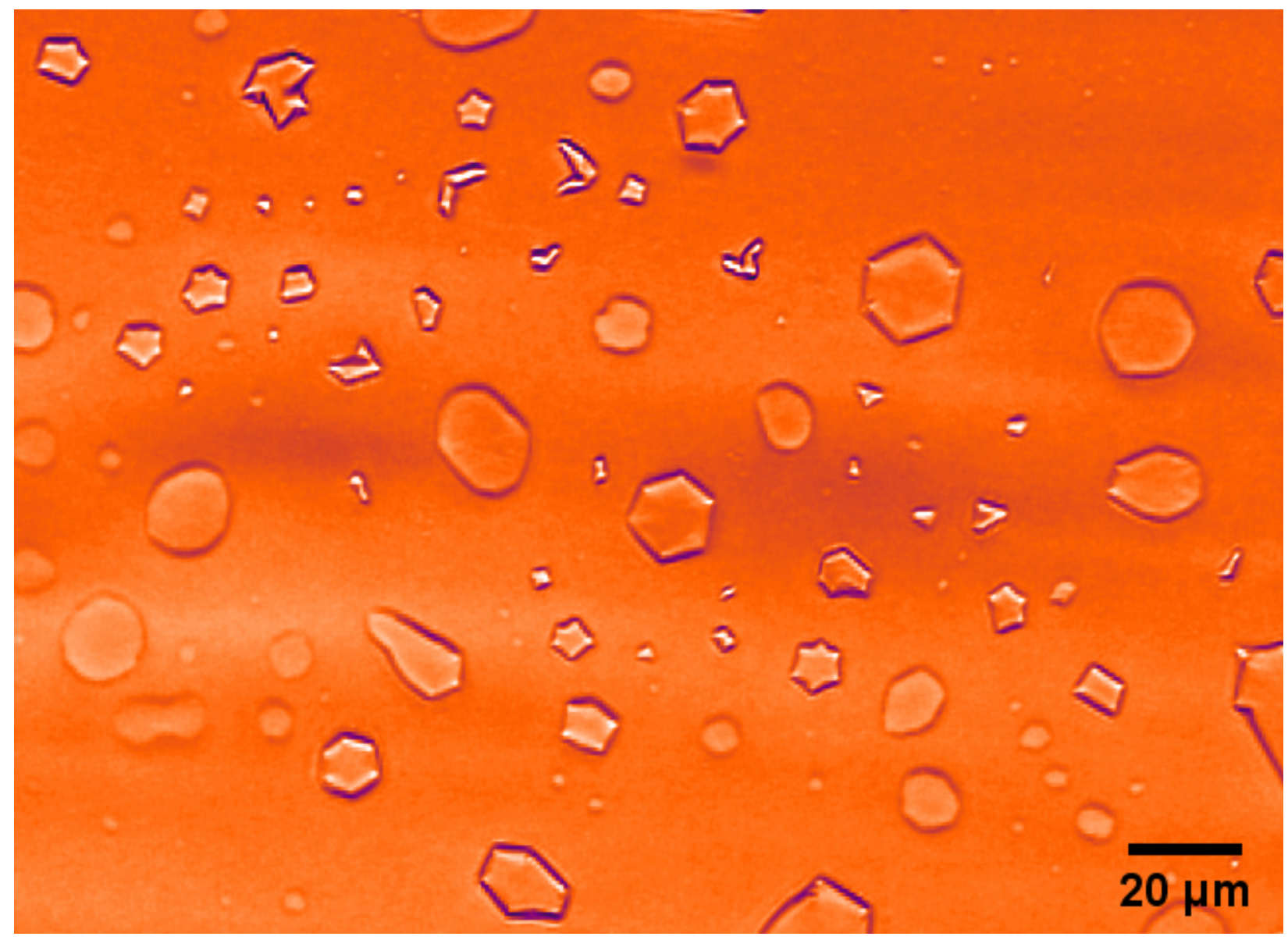

Figure S2: Water-in-oil faceted platelet-like shapes. Droplets of the aqueous $\mathrm{C}_{18} \mathrm{TAB}$ solution $(1 \mathrm{mM})$, dispersed in a $\mathrm{C}_{16} \mathrm{OH}$-containing $(1 \%) \mathrm{C}_{16}$ oil, demonstrate the formation of platelet-like faceted shapes, on cooling below the sphere-to-icosahedron transition. While most of the droplets adopt a hexagonal shape, a few other faceted shapes are observed as well: hexagrams, triangles, quadrilaterals, etc. A few droplets remain rounded, possibly dominated by wetting one of the defects in the capillary's hydrophobic coating. 


\subsection{Microscopy and imaging}

\subsubsection{Imaging setup}

Bright-field microscopy employed an inverted Nikon Ti-E microscope, with a Nikon DS-Fi1 CCD camera installed for video acquisition. The polarization microscopy mode was occasionally switched on, allowing to test for bulk freezing of the oil phase (see below). For confocal imaging, we use the Nikon A1R setup, with the illuminating wavelength set to $488 \mathrm{~nm}$. In both imaging modes, we used dry Plan Fluor 100x (NA=0.9) and Plan Apo 20X (NA=0.75) objectives, which do not touch the sample-containing capillary, thus minimizing temperature gradients within the sample. The microscopic planar interface measurements employed an Extra-Long Working Distance (ELWD) Plan Fluor 60x objective (NA=0.7).
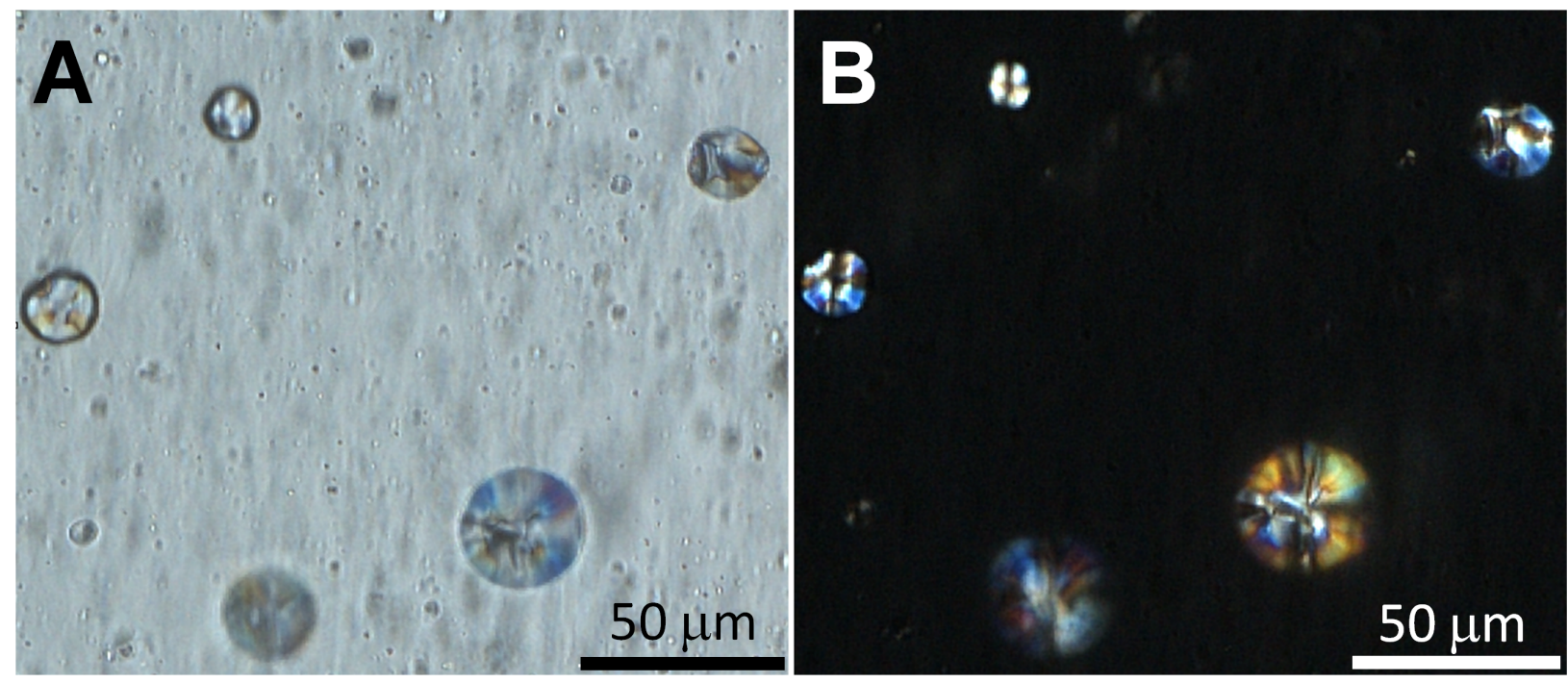

Figure S3: Bulk-frozen droplets of pure $\mathbf{C}_{16} \mathbf{O H}$ in water. Droplets of pure $\mathrm{C}_{16} \mathrm{OH}$, stabilized in water by $\mathrm{C}_{18} \mathrm{TAB}(1 \mathrm{mM})$, undergo a bulk-freezing transition at $T \approx 51^{\circ} \mathrm{C}$. The fully-frozen rotator $\left(R_{I I}\right)$ droplets are non-transparent and birefringent. Thus, they are easily distinguished, by both (A) bright-field or (B) polarization imaging, from the transparent, non-refringent, liquid droplets, where the faceting phenomena, studied here, occur. 


\subsubsection{Excluding bulk phase transformation at the faceting transition}

To confirm that no bulk phase transformation occurs in our faceted droplets, we show that such transformations, if occurred, would have been clearly visible with our setup. For this demonstration, we employ emulsions droplets of pure $\mathrm{C}_{16} \mathrm{OH}$ alcohol, stabilized in water by the $\mathrm{C}_{18} \mathrm{TAB}$ surfactant $(1 \mathrm{mM})$. These $\mathrm{C}_{16}$-free droplets do not exhibit any faceting effects, remaining spherical down to their bulk freezing temperature $\left(\sim 51^{\circ} \mathrm{C}\right)$, where they form a rotator $R_{I I}$ phase. ${ }^{2}$ The frozen droplets are non-transparent, with the birefringence effects clearly discernible even in the bright-field imaging mode (Fig. S3A). The birefringence of the $R_{I I}$ bulk is emphasized in the polarization microscopy mode, where the sample resides between two mutually-perpendicular polarizers (Fig. S3B). No birefringence is observed for the faceted droplets (either the pure $\mathrm{C}_{16}$, the mixed $\mathrm{C}_{16}: \mathrm{C}_{16} \mathrm{OH}$, or the water-in-oil ones) studied in the present work. Also, the liquid faceted droplets are transparent down to their bulk freezing temperature.

\subsection{Interferometry}

\subsubsection{Optical setup}

For the interferometry-based measurement of the three-dimensional shape of the droplets, we employed the Nikon A1R setup (see Section 1.3), with the confocal pinhole opened to its maximum. A water droplet, settled by gravity to the bottom of the sample-containing glass capillary, is illuminated from below by a $\lambda=488 \mathrm{~nm}$ laser. Both the illuminating and the reflected light pass through a dry 20X Plan Apo objective. Scanning across the focal plane employs the galvano scanning mode of our confocal system. The light reflected from the droplet's interface interferes with the light reflected from the glass bottom of the capillary, giving rise to interference fringes (see Fig. 3A of the main text). 


\subsubsection{Theoretical modeling of interferometric images}

To model the interference fringes, we simulate an icosahedral droplet ${ }^{3}$ located above the bottom of the capillary, which is taken as the $z=0$ plane. The surfaces of the icosahedron which face towards the reflecting plane are given by $z(x, y)$. The intensity of the fringes is approximated by $I(x, y)=\cos ^{2}\left(2 \pi n_{o} z(x, y) / \lambda+\pi\right) \Omega$, where $n_{0}=1.434$ is the refractive index of oil, and $\Omega$ accounts for the loss of the reflected light rays which are oriented at a too-high angle to pass through the objective. With the $20 \mathrm{X}$ objective used in these studies, only rays propagating at an angle $-(3 / 8) \pi \lesssim \theta \lesssim 3 / 8 \pi$ with respect to the optical axis, can reach the detector.

Denoting the corresponding angular range by $\Delta \theta=(3 / 4) \pi$, we obtain $\Omega=\left[\left(\Delta \theta-2 \theta_{i}\right) / \Delta \theta\right] \Theta(\Delta \theta-$ $\left.2 \theta_{i}\right)$, where $\Theta()$ is the Heaviside step function and $\theta_{i}$ is the angle between the optical axis and the $z(x, y)$ surface normal. Fig. 3B of the main text is obtained by plotting $I(x, y)$ using the 'gray' Matlab $^{T M}$ colormap. For the theoretical interferogram to match the shape of the experimental one, we varied the orientation of the model icosahedron with respect to the bottom of the capillary, achieving a good agreement with the experiment. In the above expressions, we approximate the circular aperture of the objective by a square and neglect the dependence of the intensity reflected by the icosahedron on the angle of incidence, to simplify the calculation. Taking these smaller effects into account should further improve the agreement between the experimental and the theoretical interferograms of the liquid icosahedron in Fig. 3 of the main text.

\subsection{Depletion of $\mathrm{C}_{18} \mathrm{TAB}$ in water-in-oil droplets}

\subsubsection{Adsorption to an interface between the bulk phases}

To calculate the concentration of $\mathrm{C}_{18} \mathrm{TAB}$ in the bulk of an oil-suspended droplet of $\mathrm{C}_{18} \mathrm{TAB}$ 's aqueous solution, we first estimate the adsorption of $\mathrm{C}_{18} \mathrm{TAB}$ to a liquid interface between the semi-infinite $\mathrm{C}_{16}$ oil and $\mathrm{C}_{18} \mathrm{TAB}$ aqueous solution phases, based on the data available in the literature. ${ }^{4}$ Since the adsorption data are available for $\mathrm{C}_{12} \mathrm{TAB}, \mathrm{C}_{14} \mathrm{TAB}$, and $\mathrm{C}_{16}$ TAB only (Fig. S4A), but not for the $\mathrm{C}_{18} \mathrm{TAB}$, we rescale the bulk concentrations $c$ of each of these surfactants by a constant factor $\alpha$, which renders overlapping the adsorption values $\Gamma$ for all the three $\mathrm{C}_{n} \mathrm{TAB}$ sur- 
factants (12 $\leq n \leq 16$ ) (Fig. S4B). The very good overlap between the rescaled data indicates that the mechanism of interfacial adsorption is the same for all of these surfactants. Moreover, the scaling factor $\alpha$, plotted on a semilog-y scale as a function of $n$, exhibits a simple linear variation (Fig. S4C), further confirming that the $n$-dependence of $\Gamma$ is highly systematic. Extrapolating this highly linear behavior of $\alpha$ (on a semilog scale) to $n=18$ (Fig. S4C), yields $\alpha=0.13 \pm 0.04$. Substituting this $\alpha$ into the general $\Gamma(\alpha c)$ dependence of the $\mathrm{C}_{n}$ TABs (Fig. S4B), yields the $\Gamma(c)$ dependence for the interfacial adsorption of $\mathrm{C}_{18} \mathrm{TAB}$, which is the surfactant of interest in our studies.

\subsubsection{Adsorption to an interface of a droplet of radius $R$}

With the $\Gamma(c)$ for an interface between the semi-infinite bulk phases at hand, we now evaluate the adsorption equilibrium for a $\mathrm{C}_{16}$-suspended $\mathrm{C}_{18} \mathrm{TAB}$ solution droplet of radius $R$. The total number of $\mathrm{C}_{18} \mathrm{TAB}$ surfactant molecules in this droplet, including both the bulk and the surface of the droplet, is $N_{T}=(4 / 3) \pi R^{3} c_{\infty} N_{A}$, where $c_{\infty}$ is the molarity of the macroscopic bulk solution out of which the droplet has been initially prepared, and $N_{A}$ is the Avogadro number. Once the equilibrium intra-droplet distribution of $\mathrm{C}_{18} \mathrm{TAB}$ is established, a fraction of these $N_{T}$ molecules remains in the droplet's bulk, while the rest are adsorbed at the interface. Denoting the equilibrium droplet's bulk molarity by $c_{b}$, we obtain the number of the surface adsorbed $\mathrm{C}_{18} \mathrm{TAB}$ molecules: $N_{s}=4 \pi R^{2} N_{A} \Gamma\left(c_{b}\right)$, where the possible weak dependence of $\Gamma\left(c_{b}\right)$ on the interfacial curvature is neglected. Correspondingly, the number of $\mathrm{C}_{18} \mathrm{TAB}$ molecules in the droplet's bulk is: $N_{b}=$ $N_{T}-N_{s}=(4 / 3) \pi R^{3} c_{\infty} N_{A}-4 \pi R^{2} N_{A} \Gamma\left(c_{b}\right)$. Finally, the molarity of the droplet's bulk is obtained as:

$$
c_{b}=\frac{N_{b}}{(4 / 3) \pi R^{3} N_{A}}=c_{\infty}-3 \frac{\Gamma\left(c_{b}\right)}{R} .
$$

We solve Eq. 1 graphically for a given $R$ and $c_{\infty}$. Remarkably, for any practically-relevant choice of $R$ and $c_{\infty}$, the obtained $c_{b}$ is significantly lower than the concentration of the corresponding infinite bulk solution $c_{\infty}$, as clearly seen in Fig. S4D. For example, for a $10 \mu \mathrm{m}$-radius droplet, 

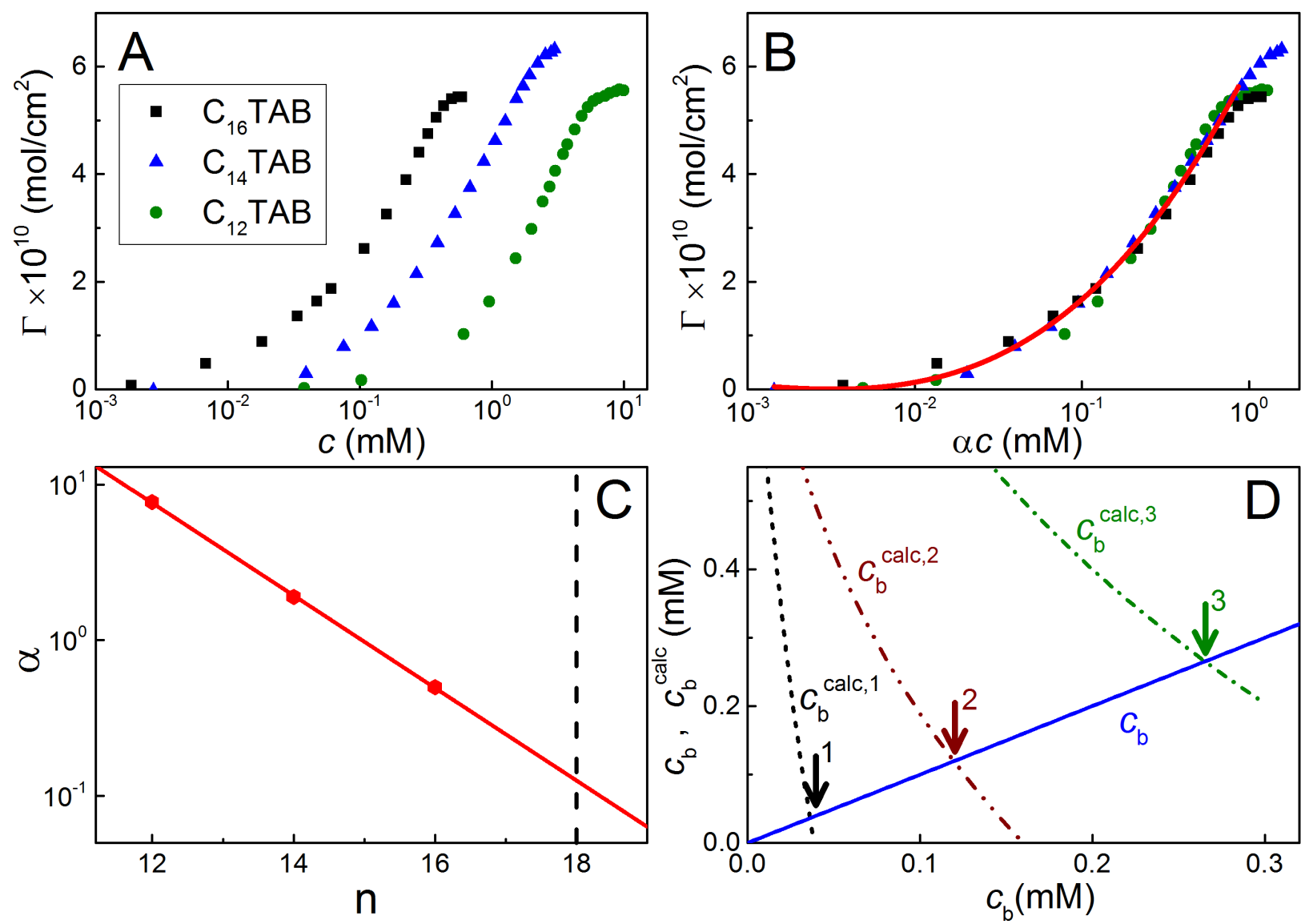

Figure S4: Estimating the bulk $\mathbf{C}_{18}$ TAB depletion in water droplets suspended in a pure continuous $\mathbf{C}_{16}$ phase. (A) The interfacial adsorption of different $\mathrm{C}_{n} \mathrm{TAB}$ surfactants (see legend), adopted from the literature. ${ }^{4}$ (B) All curves in panel A collapse onto a single curve upon rescaling the concentrations by a parameter $\alpha$, which is constant for each surfactant. (C) The $\alpha$ parameters exhibit a systematic scaling with the $\mathrm{C}_{n} \mathrm{TAB}$ carbon number $n$, providing an estimate for $\alpha(n=$ 18): the intersection of the red solid and black dash lines. (D) Graphical solutions of Eq. 1, obtained for three different parameter combinations: (1) $c_{\infty}=1 \mathrm{mM}, R=10 \mu \mathrm{m}$; (2) $c_{\infty}=1 \mathrm{mM}$, $R=20 \mu \mathrm{m}$; (3) $c_{\infty}=1.5 \mathrm{mM}, R=20 \mu \mathrm{m}$, demonstrate that the bulk $\mathrm{C}_{18} \mathrm{TAB}$ concentration of the droplets is significantly reduced. This reduction, accompanied by a consequent reduction of the $\mathrm{C}_{18} \mathrm{TAB}$ concentration at the interface, results in a significant decrease in the temperatures of the interfacial transitions. Thus, the faceting transition in $\mathrm{C}_{16}$-dispersed droplets is preempted by the bulk freezing of the oil. Here $c_{b}^{\text {calc }}$ denotes the right-hand side of Eq. 1 . 
at the high end of our $R$-scale, prepared from a $1 \mathrm{mM}$ bulk solution, we obtain $c_{b} \approx 0.04 \mathrm{mM}$. Higher bulk solution concentrations are difficult to work with due to $c$-increasing $\mathrm{C}_{18} \mathrm{TAB}$ precipitation. The interfacial freezing temperature for $c_{b} \approx 0.04 \mathrm{mM}$ is at $T \approx 20.7^{\circ} \mathrm{C}$, about $5^{\circ} \mathrm{C}$ lower than for a $1 \mathrm{mM}$ solution, as demonstrated in our earlier studies of planar interfaces (Fig. 6 in Ref. 5). Since the down-shift in $T_{d}$ should be of the same size as the downshift in the onset temperature of the interfacial freezing, we expect $T_{d} \approx 20-5=15^{\circ} \mathrm{C}$, which is far below the bulk freezing of the continuous $\mathrm{C}_{16}$ phase $\left(\sim 18^{\circ} \mathrm{C}\right)$. Thus, this calculation demonstrates that the pure- $\mathrm{C}_{16}$-dispersed droplets of the aqueous $\mathrm{C}_{18}$ TAB solution should not exhibit any faceting transitions, even in the case of negligible interfacial curvature effects. By dispersing the droplets in a $\mathrm{C}_{16} \mathrm{OH}$-containing oil, where the $\mathrm{C}_{16} \mathrm{OH}$ alcohol plays a role of a cosurfactant, we reduce the interfacial concentration of $\mathrm{C}_{18} \mathrm{TAB}$ necessary for the faceting transition to take place, allowing the transition to be observed for the micron-size droplets and for $\mathrm{C}_{18} \mathrm{TAB}$ concentrations of $1 \mathrm{mM}$ and below, as described in the main text.

\subsection{Size dependence of water droplets' faceting transitions}

The surface-to-volume effects, discussed in Section 1.5.2, play an important role in determining the $T_{d}$ values of water droplets in $\mathrm{C}_{16} \mathrm{OH}$-free $\mathrm{C}_{16}$ oil. However, the addition of $\mathrm{C}_{16} \mathrm{OH}$ to the oil significantly weakens these effects. Thus, a reverse dependence on $R$ sets in, with the small droplets undergoing the faceting transition at a higher temperature (black squares in Fig. S5). Remarkably, a similar dependence was previously observed for the droplets of pure $\mathrm{C}_{16}$-alkane, suspended in aqueous $\mathrm{C}_{18} \mathrm{TAB}$ solutions (open blue circles in Fig. S5). ${ }^{3}$ This (weak) size dependence, which is currently under an active investigation ${ }^{6-8}$, may possibly result from either a variation in the surface-lattice dislocation concentration, or from a curvature-induced variation in the interfacial surfactant concentration. In any case, the very similar behavior observed for both the water droplets and for the $\mathrm{C}_{16}$-oil ones, supports our claim for the similarity of these two systems. This similarity is not explained by the mechanism which attributes the faceting to oil confinement effects, while it provides a strong support for our interfacially-driven faceting mechanism. 


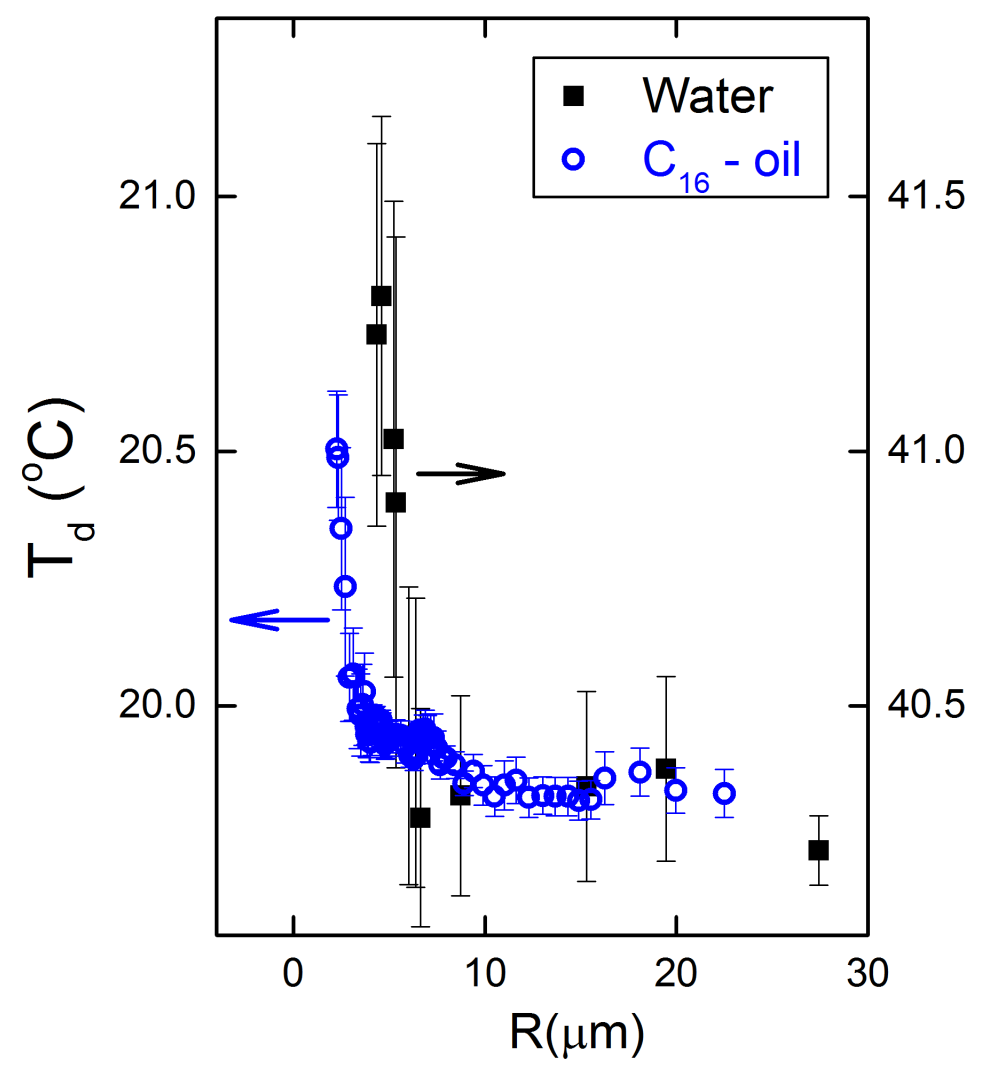

Figure S5: The faceting transition temperature $T_{d}$ as a function of the droplet size $R$. A similar behavior is observed for both the water-in-oil droplets (black squares) and the $\mathrm{C}_{16}$-oil-inwater droplets (open blue circles). 


\subsection{Interfacial tension measurements}

\subsubsection{Experimental setup}

The Wilhelmy plate method, well documented in the literature ${ }^{1,3,9-11}$, was used to measure the interfacial tension of the planar buried oil-water interfaces, employing a glass plate of 41-mm circumference. For the Wilhelmy plate measurements, the sample, contained in a glass beaker, was placed into a two-stage oven, with an inner temperature-controlled $\left( \pm 0.01^{\circ} \mathrm{C}\right)$ cell, and an outer aluminum cell employed for thermal isolation. The interfacial tension was measured by an electronic Precisa ${ }^{T M}$ semi-micro balance, and recorded by a computer, with 20 measurements averaged at each temperature. The temperature was typically scanned in steps of $0.1^{\circ} \mathrm{C}$, with the waiting time after each step being 30s. The Wilhelmy plate and all the glassware contacting the sample are cleaned with a fresh Piranha solution before each sample.

\subsubsection{Interfacial entropy}

The relation between the entropy of interface formation and $\gamma(T)$, is discussed extensively in the literature. ${ }^{1,3,10-12}$ Therefore, we only note that the change in $d \gamma(T) / d T$ upon interfacial freezing at $T_{s}, \Delta\left(d \gamma\left(T_{s}\right) / d T\right)=d \gamma\left(T<T_{s}\right) / d T-d \gamma\left(T>T_{s}\right) / d T$, yields the change in the interfacial entropy upon the interfacial freezing: $\Delta S \equiv S\left(T<T_{s}\right)-S\left(T>T_{s}\right)$. As $d \gamma / d T$ is (roughly) constant both below and above $T_{s}$, the only significant change in this quantity occurs at $T_{s}$ (see Fig. 2A of the main text). Since the interfacial coverage of the surfactant does not significantly change ${ }^{13-15}$ at $T=T_{s}$, the main contribution to $\Delta S$ is due to the freezing of the interfacial layer's alkyl chains. While the alkyl tails of the $\mathrm{C}_{18} \mathrm{TAB}$ surfactants are longer than these of the $\mathrm{C}_{16}$-alkane by two $\mathrm{CH}_{2}$ groups, this change corresponds to only a minor $\sim 10 \%$ increase in the surfactant's entropy. Since $\mathrm{C}_{18} \mathrm{TAB}$ only covers a fraction of the interface, with the rest being covered by either the $\mathrm{C}_{16}$ alkane or the $\mathrm{C}_{16} \mathrm{OH}$ alcohol, the influence of the slightly-longer alkyl tail 
of $\mathrm{C}_{18} \mathrm{TAB}$ is much smaller than the sensitivity of our measurements. Thus, in order to estimate the number of frozen monolayers covering the interface, we divide the experimentally-measured $\Delta S$ (entropy loss, per unit area of the interface) by the known entropy loss on freezing of a pure- $\mathrm{C}_{16}$ interfacial layer, $0.896 \mathrm{mN} / \mathrm{m} \mathrm{K}$, available in the literature. ${ }^{11}$ This calculation yields the number of interfacially-frozen layers as $\approx 1,1.5$, and 0.6 for the concentration of $\mathrm{C}_{16} \mathrm{OH}$ in the oil phase being $0,0.18$, and $0.4 \%$, respectively (see the inset to Fig. $2 \mathrm{~B}$ of the main text). The higher $\Delta S$

obtained for the $\mathrm{C}_{16} \mathrm{OH}$-containing $(\sim 0.2 \%)$ samples suggests either a partial freezing of a second interfacial layer, or an increase in the interfacial order due to the possible formation of intra-layer $\mathrm{OH}-\mathrm{OH}$ hydrogen bonds. Studies by other methods, potentially allowing to decide between these two scenarios are currently underway.

\section{Supporting Videos' Captions}

Video 1. A cooling scan (at $0.5^{\circ} \mathrm{C} / \mathrm{min}$ ), demonstrating water-in-oil droplets undergoing a sphereto-icosahedron transition. The video shows the scan from time $03 \mathrm{~m}: 10$ s (see label at the bottomright side), when $T=41.4^{\circ} \mathrm{C}$. Some of the transitions are marked by black arrows, with the movie temporarily paused. Notably, in this sample we observe the droplets to undergo periodic sphereto-icosahedron-to-distorted-to-sphere transition sequences, instead of the icosahedra irreversibly deforming into the platelet-like faceted shapes. Future studies should allow the origin for these two distinct droplet behavior patterns to be resolved. Here the dispersed phase is a $1 \mathrm{mM} \mathrm{C}_{18} \mathrm{TAB}$ solution, and the continuous phase is a $1.3 \%(\mathrm{w} / \mathrm{w})$ mixture of $\mathrm{C}_{16} \mathrm{OH}$ in $\mathrm{C}_{16}$.

Video 2. An icosahedral water-in-oil droplet distorting on cooling into an (almost-) plateletlike shape, as observed in the interferometric imaging mode. The imaging in this mode employs laser light reflection (see Section 1.4 of the Supplementary Material). In this mode, only the parts of the droplet which are at a relatively small angle to the bottom of the sample-containing capillary back-reflect the light into the objective. Thus, initially, only a small portion of the droplets' top is visible. As the droplet undergoes a flattening transition, larger portions of its surface become visible. Interference fringes, clearly visible in the central part of the droplet, indicate a gradual 
flattening of its faceted shape. The droplet is composed of a $1 \mathrm{mM}$ aqueous $\mathrm{C}_{18} \mathrm{TAB}$ solution; the continuous phase is a $0.6 \%(\mathrm{w} / \mathrm{w})$ mixture of $\mathrm{C}_{16} \mathrm{OH}$ in $\mathrm{C}_{16}$.

Video 3. An interface between semi-infinite bulk phases of oil and water undergoes shape and wrinkling transitions, during a cooling scan (at $\left.0.6^{\circ} \mathrm{C} / \mathrm{min}\right)$. These observations prove that the self-shaping transitions take place even for the very-low-curvature interface separating two bulk phases. Here the aqueous phase (at the bottom left) is a $1 \mathrm{mM} \mathrm{C}_{18} \mathrm{TAB}$ solution; the oil phase (top-right) is a $0.4 \%(\mathrm{w} / \mathrm{w})$ mixture of $\mathrm{C}_{16} \mathrm{OH}$ in $\mathrm{C}_{16}$.

Video 4. A stack of confocal slices across a wrinkled interface at $T=26.9^{\circ} \mathrm{C}<T_{S E}$. The slices' orientation with respect to the capillary is shown in Fig. 4A of the main text. The height above the bottom of the stack is labeled at the bottom of the screen. Some of the wrinkles are marked by white arrows. The sample composition is the same as for Video 3, but with the oil phase fluorescently-labeled.

\section{References}

(1) Guttman, S.; Sapir, Z.; Ocko, B. M.; Deutsch, M.; Sloutskin, E. Temperature-tuned faceting and shape-changes in liquid alkane droplets. Langmuir 2017, 33, 1305-1314.

(2) Berwanger, R.; Henschel, A.; Knorr, K.; Huber, P.; Pelster, R. Phase transitions and molecular dynamics of n-hexadecanol confined in silicon nanochannels. Phys. Rev. B 2009, 79, 125442.

(3) Guttman, S.; Sapir, Z.; Schultz, M.; Butenko, A. V.; Ocko, B. M.; Deutsch, M.; Sloutskin, E. How faceted liquid droplets grow tails. Proc. Natl. Acad. Sci. U.S.A. 2016, 113, 493-496.

(4) Medrzycka, K.; Zwierzykowski, W. Adsorption of alkyltrimethylammonium bromides at the various interfaces. J. Colloid Interf. Sci. 2000, 230, 67-72.

(5) Sloutskin, E.; Bain, C. D.; Ocko, B. M.; Deutsch, M. Surface freezing of chain molecules at the liquid-liquid and liquid-air interfaces. Faraday Discuss. 2005, 129, 339-352. 
(6) Guttman, S.; Kesselman, E.; Jacob, A.; Marin, O.; Danino, D.; Deutsch, M.; Sloutskin, E. Nanostructures, faceting, and splitting in nanoliter to yoctoliter liquid droplets. Nano Lett. 2019, 19, 3161-3168.

(7) García-Aguilar, I.; Fonda, P.; Giomi, L. Dislocation screening in crystals with spherical topology. arXiv 2020, cond-mat.soft, arXiv:2002.02446v1.

(8) Haas, P. A.; Cholakova, D.; Denkov, N.; Goldstein, R. E.; Smoukov, S. K. Shape-shifting polyhedral droplets. Phys. Rev. Research 2019, 1, 023017.

(9) Wu, X. Z.; Ocko, B. M.; Sirota, E. B.; Sinha, S. K.; Deutsch, M.; Cao, G. H.; Kim, M. W. Surface Tension Measurements of Surface Freezing in Liquid Normal Alkanes. Science 1993, $261,1018-1021$.

(10) Tamam, L.; Pontoni, D.; Sapir, Z.; Yefet, S.; Sloutskin, E.; Ocko, B. M.; Reichert, H.; Deutsch, M. Modifcation of deeply buried hydrophobic interfaces by ionic surfactants. Proc. Natl. Acad. Sci. U.S.A. 2011, 108, 5522-5525.

(11) Ocko, B. M.; Wu, X. Z.; Sirota, E. B.; Sinha, S. K.; Gang, O.; Deutsch, M. Surface Freezing in Chain Molecules: Normal Alkanes. Phys. Rev. E 1997, 55, 3164-3182.

(12) Motomura, K. Thermodynamic Studies on Adsorption at Interfaces. I. General formulation. J. Colloid Interf. Sci. 1978, 64, 348-355.

(13) Lei, Q.; Bain, C. D. Surfactant-Induced Surface Freezing at the Alkane-Water Interface. Phys. Rev. Lett. 2004, 92, 176103.

(14) Wilkinson, K. M.; Qunfang, L.; Bain, C. D. Freezing transitions in mixed surfactant/alkane monolayers at the air-solution interface. Soft Matter 2005, 2, 66.

(15) Sloutskin, E.; Sapir, Z.; Bain, C. D.; Lei, Q.; Wilkinson, K. M.; Tamam, L.; Deutsch, M.; Ocko, B. M. Wetting, mixing, and phase transitions in Langmuir-Gibbs films. Phys. Rev. Lett. 2007, 99, 136102. 